\title{
Recent Progress in the Diagnosis and Precise Nanocarrier-Mediated Therapy of Inflammatory Bowel Disease
}

\author{
Liucan Wang \\ Min Yu iD \\ Hua Yang \\ Department of General Surgery, Xinqiao \\ Hospital, Army Medical University, \\ Chongqing, People's Republic of China
}

\begin{abstract}
The effective colon drug delivery remains to be an international frontier research in inflammatory bowel disease (IBD) therapy. The exploration and research of nanocarrierbased nanomedicine with great potential brings new opportunities for IBD therapy and diagnoses. Functional nanocarriers with varying morphology and characteristics can not only effectively avoid the destruction of the complex gastrointestinal (GI) tract microenvironment but also endow drugs with target therapy and improved bioavailability, thus elevating therapeutic efficacy. In this review, we illustrated several challenges in IBD therapy, then emphasis on some latest research progress of nanoparticles based therapy of oral administration, rectal administration and parenteral administration, as well as IBD diagnoses. Finally, we described the future perspective of nanocarriers in the treatment and diagnoses of IBD.

Keywords: inflammatory bowel disease, gastrointestinal tract microenvironment, drug delivery system, precise therapy, diagnose
\end{abstract}

\section{Introduction}

Inflammatory bowel disease (IBD), exemplified by Crohn's disease (CD) and ulcerative colitis (UC), is a chronic idiopathic inflammatory condition that is associated with an increased risk of colorectal cancer. ${ }^{1,2}$ Due to the multifarious etiology and complexity of IBD, an effective treatment strategy for IBD remains an urgent need and scientific issue. The primary therapeutic goals of UC treatment are to improve patients' quality of life by maintaining clinical remission and reducing treatment complications. Clinical therapeutics include 5-aminosalicylic acid derivatives (eg, sulfasalazine), glucocorticoids (eg, prednisone), and immunosuppressants (eg, tacrolimus, azathioprine and mercaptopurine) that have achieved considerable success. ${ }^{3}$ Nevertheless, conventional therapeutics remains a major challenge for further clinical application. The potential reasons for this are as follows: conventional drug-administration modalities, such as oral administration, intravenous administration, and rectal administration of therapeutic agents, are usually accompanied by limited therapeutic efficacy, inevitable adverse effects and/or dependency. ${ }^{4,5}$ In addition, these therapeutic agents are not selective, which results in nonspecific distribution, particularly in blood vessels, liver and kidney. Notably, extended overuse can lead to the development of multidrug resistance (MDR). ${ }^{6,7}$ In addition to the aforementioned challenges, the short half-life and low bioavailability of biological agents strictly limit their therapeutic effects to a large extent. ${ }^{8}$ Therefore, the critical goal of enhanced IBD therapy is
Correspondence: Min Yu; Hua Yang $\mathrm{Tel} / \mathrm{Fax}+8623-687-55705$ Emailyumimianbao@I63.com; huayang@tmmu.edu.cn 
bypassing the complex gastrointestinal tract (GIT) microenvironment to achieve targeted drug delivery, maintain drug activity and maximize drug availability. ${ }^{9}$

Because of bottlenecks in traditional clinical IBD therapy, many studies have been conducted to improve the therapeutic strategies more be more efficient and safe. ${ }^{10}$ Nanomedicine-based drug delivery systems (DDSs), as products of emerging technology with unique drug delivery properties, have expanded the opportunities for IBD therapy and, to some extent, have paved a new avenue to solve the aforementioned problems. ${ }^{11}$ Nanoparticles are composed of new types of material and range from 1 to $100 \mathrm{~nm}$ in diameter, and nanoparticle-based DDSs have different shapes, structures, and sizes. Therapeutic agents can be loaded to nanoparticles by encapsulation, surface attachment, or entrapment. ${ }^{12}$ For decades, the DDSs such as lipid nanoparticles, ${ }^{13}$ polymer nanoparticles,${ }^{14}$ mesoporous nanoparticles (eg, MSN, MPDA), ${ }^{15}$ chitosan nanoparticles, ${ }^{16}$ hydrogel nanoparticles ${ }^{17}$ and metal nanoparticles ${ }^{18}$ have been used in delivering therapeutics for managing disease (Figure 1). The goal of nanomedicine-mediated IBD therapy is to protect drugs from premature release and degradation to achieve targeted accumulation in the inflamed area. Nanoparticles have a large surface area, tunable size and modifiable surface, providing opportunities for achieving treatment goals. ${ }^{19}$ More importantly, particularly small nanoparticles are endowed with the ability to permeate the mucosa layer efficiently and enter individual intestinal epithelial cells $^{20}$ and thus achieve passive targeting via enhanced epithelial permeability and retention (eEPR) effects in the inflamed area of the colon. ${ }^{21}$ Therefore, the advantages of DDSs include high drug-loading capacity, efficient drug protection, targeted drug delivery, controlled drug release, excellent biocompatibility and reduced dosage frequency, effectively improving the bioavailability and therapeutic efficiency of drugs. ${ }^{22}$

Nevertheless, the mechanism of targeted drug delivery mainly depends on the physiological conditions of the inflammation area and the corresponding functional design of the nanoparticles. DDSs, often endowed with the capacity for $\mathrm{pH}$-induced accumulation, ${ }^{23}$ charge-mediated interaction, reactive oxygen species (ROS)-mediated accumulation, ${ }^{24}$ (glutathione) GSH-mediated accumulation, enzyme-mediated accumulation (Figure 2) and other effects, have been successfully developed and adopted for oral drug delivery.

Therefore, this article comprehensively reviews the recent progress of nanoparticle-based IBD therapy. First, the major challenges associated with drug delivery are summarized. Then, the accumulation properties of drug-loaded nanoparticles in the inflamed colon, including passive targeting and active targeting are depicted, and the principles of nanoparticle application strategies are highlighted. Further, we focus on the recent advancements of nanocarrier-based treatment strategies based on active targeting, passive targeting and inflamed colon-activated nanotherapy, including $\mathrm{pH}$ , ROS-, GSH- and enzyme-based activated nanotherapies, and the application of nanoparticles based diagnose was depicted. Finally, the prospects and challenges in the rapidly growing field of nanoparticle-based IBD therapy are discussed, including their expected future applications.

\section{Major Challenges in Nanocarrier-Based IBD Therapy}

Among the different routes of administration of the medications, patient compliance cannot be overemphasized. Although oral administration is considered the best choice, offering patient convenience for long-term use, ${ }^{25}$ it must be recognized that drugs administered through this route are inevitably exposed to the complex GIT microenvironment, and drug absorption and utilization are thus affected. Similarly, nanoparticle-based DDSs face a series of challenges that hinder the transport and absorption of drugs, reducing their efficacy, similar to the effects of intravenous and rectal administration. Moreover, for small molecules drugs in traditional medicine, when administered alone, many exhibit low bioavailability, let alone biological
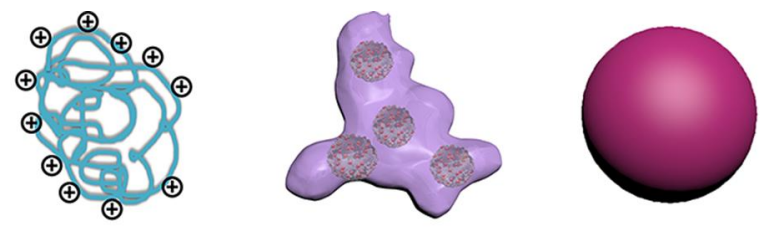

Chitosan nanoparticles

Metal nanoparticles

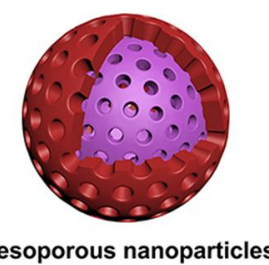

Figure I Nanoparticles that is suitable for drug delivery, including lipid nanoparticles, polymer nanoparticles, mesoporous nanoparticles, chitosan nanoparticles, hydrogel nanoparticles and metal nanoparticles. 


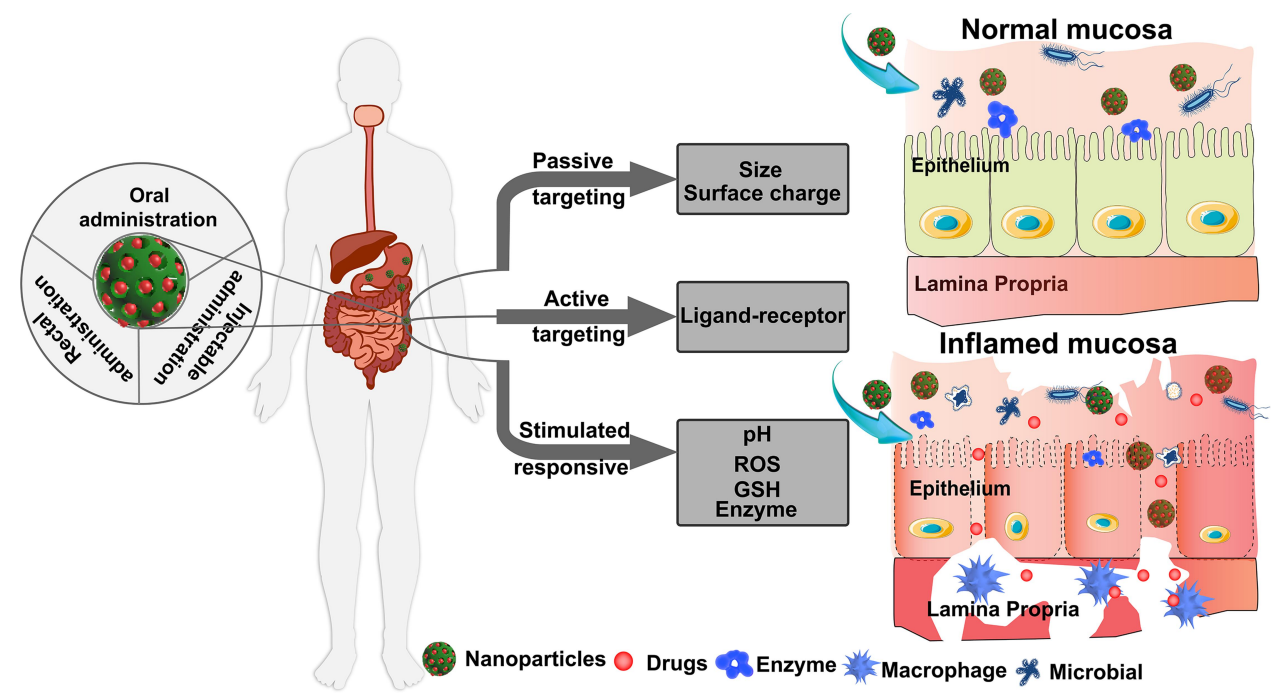

Figure 2 Schematic illustration of GIT microenvironment-based target drug delivery.

agents. In this section, some of the main challenges to nanotherapy are depicted.

\section{The GIT pH and Enzyme}

The $\mathrm{pH}$ in different GIT regions, including the inflamed areas, show significant differences, from the highly acidic stomach $(\mathrm{pH} 1.0-2.5)$ to the neutral or slightly alkaline duodenum $(\mathrm{pH} 6.0)$ to the microalkaline jejunum and ileum ( $\mathrm{pH}$ 6.0-7.5), which must be considered in drug delivery. ${ }^{25,26}$ Specifically, gastric acid is the main challenge to oral administration. Therapeutic agent exposure to acidic microenvironments can lead to hydrolysis, oxidation or deamidation of the biological agents, leading to deactivation. ${ }^{27}$ In particular, $\mathrm{pH}$ changes are likely to affect the composition of the colonic microbiome and thus the charge of the mucus.

In addition to $\mathrm{pH}$, the complex enzyme environment in the GIT is a major challenge. Gastric enzymes (mainly pepsin) and other digestive enzymes (hydrolase, lipase, peptidase and amylase) are possible causes of drug instability, and therapeutic agents such as biological agents are susceptible to degradation by various digestive enzymes in the GIT. ${ }^{28}$ Multifunctional DDSs, with their unique drug delivery advantages, are favored in the field of IBD therapy research because they are not prone to acid degradation or have anti-enzymatic activity. Previous studies have demonstrated that triglycerol monostearatebased hydrogels and ascorbyl palmitate (AP) nanoparticles can efficiently protect drugs from being degraded by enzymes. $^{17}$ Overall, multifunctional DDSs contribute a new strategy to IBD therapy by their ability to avoid enzyme degradation in the gastrointestinal tract.

\section{Intestinal Mucosa}

Notably, intestinal mucosa should also be considered. The intestinal mucosa is a negatively charged, ${ }^{29}$ highly viscoelastic, adhesive and thick $(830 \pm 110 \mu \mathrm{m})$ layer that covers the mucosal surface of the GIT, ${ }^{30}$ which greatly influences the targeted accumulation of drugs. Notably, most guest molecules are cleared in different ways and thus cannot penetrate the mucosa to enter into the systemic circulation, which significantly reduces the amount of drug that crosses the epithelial layer of the gastrointestinal tract. Therefore, the design of drug delivery systems for mucosal permeation (eg, surface modification) has recently attracted considerable attention among researchers. ${ }^{29}$ To date, the exact mechanism of DDS mucus adhesion has not been fully understood, but it is generally believed that due to interpenetration of mucus macromolecules and mucosa, specific interaction types, including electrostatic, hydrogen bond and hydrophobic interactions, are established. $^{31}$

In addition, the replacement of colonic mucin carbohydrate by sulfate and sialic acid residues results in a large number of negative charges on the colonic mucin surface. Therefore, drug carriers based on charge interactions entering the inflamed bowel provide broad convenience for drug delivery. Nanoparticles attached to the mucosa promote better contact with the surface of the mucosa, thus promoting the absorption of particles and showing better 
drug release behavior. This innovative utilization of the intestinal mucosal microenvironment provides a new way to transport drugs effectively for the treatment of IBD.

\section{Colonic Microbiome}

We appreciate the importance of the intestinal microbiome in GI physiology. The natural microflora of the colon contains metabolically active bacteria and fungi that are closely related to the host's epithelial cells and mucosal immune system. ${ }^{32}$ Although some studies have shown that the internal state of these bacteria is relatively stable in IBD, the increase in microbial species, such as Bacteroides, Eubacteria, and Streptococcus, often leads to a decrease in diversity, which has a negative impact on the stability of other populations. ${ }^{33}$

Additionally, changes in the microbial composition and function in IBD often lead to enhanced immune stimulation/ mucosal permeability, as well as epithelial dysfunction. Thus, the impaired gut barrier in IBD allows GIT bacteria to penetrate the intestinal wall, causing inflammation by immune cells, and limited treatments available to prevent this cascade are limited. The results of gut impairment in a mouse model showed that the prolonged intestinal water secretion resulted in a decrease in colonic transit time and a change in colonic microflora. ${ }^{34}$ Therefore, the drug transit time in IBD patients may be twice as long as that in healthy individuals, which makes it difficult to target specific areas of the intestine with conventional drugs.

\section{Drug Efflux at the Cellular Level}

Finally, at the cellular level, the excessive use of therapeutic agents can lead to MDR of target cells. The overexpression of the membrane protein P-glycoprotein (P-gp) encoded by MDR1 gene in the basolateral-to-apical direction across epithelium has been identified to be one of the most relevant causes of MDR. ${ }^{6}$ This glycoprotein belongs to the ATPbinding cassette $(\mathrm{ABC})$ family 1 , it can act as an ATPdependent drug efflux pump to pump a variety of structurally and pharmacologically unrelated neutral and positively charged hydrophobic compounds out of cells and reduce intracellular accumulation. ${ }^{35,36}$ The overexpression of the P-glycoprotein in epithelium limited the bioavailability of therapeutic agents to a large extent. ${ }^{37}$ In addition to P-gp, MRP2 and BCRP expressed on the apical membrane of intestinal are also epithelia efflux transporters, which transport relatively hydrophilic compounds. ${ }^{38}$ Therefore, the drug selection and design strategy of appropriate drug carriers will pave a new path for the treatment of IBD.
As discussed in this section, the change in the GIT microenvironment caused by different physiological and pathological factors is the main reason for poor drug efficacy. By analyzing these factors, DDS-based protective and targeted drug delivery strategy may be practical and effective for IBD nanotherapy.

\section{Different Administration Methods}

As previously depicted, there are different administration methods in IBD therapy. Compared with oral administration, intravenous (I.V.) injection provides high systemic bioavailability and reduces the first-pass metabolism of drugs. However, for intravenous (I.V.) injection, blood circulation is the first step for DDSs in the journey, the phagocytosis of macrophages is inevitable. Moreover, blood is a very complex liquid, including salt, sugar, protein, enzymes and amino acids and other substances, which make the instability of nanocarriers caused by aggregation and embolism. ${ }^{39}$ Intravenous (I.V.) injection provides high systemic bioavailability and reduces first-pass metabolism of drugs. However, the high systemic bioavailability inevitably increases the possibility of severe drug side effects. Rectal administration offers topical delivery and provides local high concentrations of drugs for the distal colonic, thus leading to lower systemic drug exposure. ${ }^{40}$ For rectal administration, poor drug retention and limited medication adherence remain challenging; this topical therapy may have problems with leakage, retention, and bloating. ${ }^{41}$ Nevertheless, in chronic gastrointestinal diseases that require long-term medication, injectable administration of drugs is less desirable than oral administration, as injections are associated with pain and require specialized personnel for their administration. Therefore, oral drug delivery strategy is preferred. For the efficient use of some biological agents, we believe that the design of nanocarriers will break the bottleneck and have good potential in IBD therapy applications. With the development of nanotechnology, DDSs can protect drugs from damage and enable them to anchor specifically to the sites of disease.

\section{Passively and Actively Targeted Drug Delivery}

In general, therapeutic agents delivered in vivo should be protected and their premature release should be prevented to ensure that the drug accumulates in the inflamed area of the colon to the maximum extent, thereby selectively relieving intestinal inflammation without affecting normal tissues. For example, with comprehensive studies on the 
cellular and molecular mechanisms of the IBD pathogenesis, a growing number of biologics, such as antitumor necrosis factor receptor (TNF- $\alpha$ ), nucleic acid receptor fragments and monoclonal antibodies, have been developed. $^{42-44}$ Unfortunately, the biologics often leads to nonspecific distribution, causing a variety of adverse reactions and reduced compliance, and the drugs are probably degraded by various digestive enzymes in the GIT. $^{27,45}$ The complex GIT microenvironment is a threat to the bioavailability of biologics, thus limiting the efficacy of oral administration. ${ }^{46,47}$ Therefore, to maximize the therapeutic effect, it is essential to develop innovative drug delivery strategies to protect drug activity.

Fortunately, with the development of multifunctional targeting strategies, DDSs seem likely candidates to meet these strategic goals. In general, targeting methods are classified into two categories, namely, active targeting methods and passive targeting methods. ${ }^{48}$ The mechanism by which a drug is targeted for delivery to the inflamed area mainly depends on the microenvironment of the inflamed colon and the corresponding functional modification. For instance, the physical properties of DDSs, such as particle size, are crucial factors in the targeted accumulation of drugs. ${ }^{20}$ In contrast, active targeting involves surface functionalization of the excipient with target-specific ligands and/or receptors to increase its selectivity. ${ }^{49}$ Therefore, targeted drug delivery can lead to more-precise spatial positioning, improve the therapeutic effect of the delivered drugs, overcome the aforementioned challenges and minimize adverse effects on healthy tissues. ${ }^{50}$ Therefore, in this section, nanotechnology-mediated targeted drug delivery, including passive and active targeting, is discussed in detail.

\section{Passive Targeting}

Despite the continuous innovative design of the DDs, poor intestinal accumulation and permeability are major challenges in the application of the DDs. ${ }^{51,52}$ As explained above, eEPR has an effect in the inflamed area of the intestine. Due to the increased permeability of the vessels and epithelium in of IBD, nanocarriers with sizes from 10 to $200 \mathrm{~nm}$ can accumulate and penetrate through the intestinal mucosa through the eEPR effect, thereby increasing the residence time in the target area and improving the efficacy of the drug. ${ }^{53-55}$ This effect is based on the histopathological abnormalities of colon tissue, such as the loss of barrier functions caused by the rupture of the intestinal epithelial cells, the increase in epithelial permeability, and the obvious infiltration of inflammatory cells into the mucosa. ${ }^{56}$

Notably, as we all know, the toxicity and side effects of metal nanoparticles such as those consisting of $\mathrm{Au}$ and $\mathrm{Ag}$ elements with small particle sizes cannot be ignored. Metal nanoparticle-based composite DDSs are designed and developed to increase biocompatibility. ${ }^{18}$ For example, $\mathrm{Au}$ nanoparticles coated with tiopronin and smaller than $10 \mathrm{~nm}$ were introduced to verify the treatment efficiency. ${ }^{55}$ Compared with nanoparticles larger than 10 $\mathrm{nm}$, the system with the smaller nanoparticles can be absorbed by intestinal epithelial cells faster and diffuse through the interior of the cells. This drug-loading system has been shown proven to inhibit the production of ROS, scavenge free radicals, and improve the antioxidant enzyme activity in diabetic mice. Considering the effect of eEPR in IBD and the ROS-scavenging characteristics of the system, a better combined therapeutic effect can be obtained.

In addition to size dependency, surface characteristics such as surface charge and surface chemistry are also crucial to passive targeting and may enhance the accumulation of smaller nanoparticles. For oral administration, positively charged DDSs are vulnerable to elimination by enzymes or may physically attach to the mucosa via charge interactions. ${ }^{30}$ Therefore, surface charge inversion of nanoparticles by controlled modification such as chitosan-based lipid nanoparticles is a creative strategy. ${ }^{16}$ A previous study demonstrated that positively charged proteins (including transferrin, ${ }^{57}$ eosinophil cationic protein $^{58}$ and bacteriostatic protein ${ }^{59}$ ) accumulate in the inflamed colon. Therefore, the positively charged surface of damaged permeable epithelium provides a target for drug carriers with a negative surface charge, ${ }^{57}$ which is a very useful strategy for passive targeting-mediated drug delivery. Research related to the negatively charged surface design of nanoparticles for accurate therapy of the inflamed colon has become a research hotspot. PEGylation with neutral to slightly negative net charge is a common surface modification, ${ }^{44,60}$ and the surface coating of negatively charged heparin (HEP), hyaluronic acid (HA), ${ }^{61,62}$ and nanozymes ${ }^{63}$ can also increase the accumulation of nanoparticles with positively charged proteins on the inflamed mucosa. ${ }^{64}$ For instance, nanozyme-based nanoparticles with a negative surface charge showed enhanced passive targeting and inflammation relief. Zheng et al designed and prepared a manganese Prussian blue nanozyme (MPBZ) system with multienzyme activity by 
mixing $\mathrm{Mn}^{2+}$ ions with polyvinylpyrrolidone (PVP) and $\left[\mathrm{Fe}(\mathrm{CN})_{6}\right]^{4-}$ ion sources with PVP. ${ }^{63}$ Nanosystems with negative surface charges can effectively accumulate in inflamed colonic mucosa, and MPBZs can act as ROS nanoscavengers. In vivo experiments showed enhanced accumulation of DDSs at the inflamed mucosa in mice with DSS-induced colitis.

In addition to surface charge modification, nanocarriers with a negative charge itself also have potential for use in passively targeted accumulation. These nanocarriers not only simplify the process of charge modification but also have a high drug-loading capacity. Zhang et al designed and constructed a negatively charged mesoporous carbon nanoparticle-based antimicrobial peptide (MDC)-loaded MDC@MCN system, ${ }^{65}$ and the excellent stability of the MCNs protected the MDCs delivered to the GIT from degradation. Combined with a negatively charged surface, this DDS greatly improved targeted enrichment efficiency at the inflammatory site in the intestine. As expected, oral administration of MDC@MCNs significantly ameliorated UC by reducing inflammation and oxidative stress, maintaining the intestinal mucosal barrier, and regulating the intestinal flora, with efficacy and safety superior to that of MDC.

In addition, clinically approved naturally negatively charged montmorillonite (MMT), which has been clinically used, can also be effectively used in charge interaction-mediated passive accumulation. Zhao et al designed and synthesized a nanozyme system of $\mathrm{CeO}_{2} @ \mathrm{MMT}$, which is capable of oral administration. ${ }^{66}$ The MMT sheets can effectively avoid systemic absorption of $\mathrm{CeO}_{2}$ and enhance the accumulation of the DDSs in inflamed sites through charge interactions. $\mathrm{CeO}_{2}$ can impart MMT with anti-ROS capabilities, and thus inflammation can be alleviated. The designed $\mathrm{CeO}_{2} @ \mathrm{MMT}$ nanozyme system with effective targeted drug delivery and ROS-scavenging activity will inspire novel strategies for developing nanomedicine in the future. Taking full advantage of the unique positive charge characteristics of inflammatory sites, NDDSs based on charge interactions to enhance accumulation have been developed and have shown excellent therapeutic effects.

Due to the intrinsic properties of rosmarinic acid (RA) with poor water solubility and low bioavailability, Chung et al developed PEGylated RA-derived nanoparticles (RANPs) with a diameter of $63.5 \pm 4.0 \mathrm{~nm} .{ }^{67}$ The PEGylated bioactive compound-derived nanoparticles not only achieved $100 \%$ RA loading capacity ${ }^{68,69}$ but also achieved safer and longer RA circulation in the blood after intravenous injection. Therefore, RANPs can prolong the biological activity and have a higher probability of accumulating in the inflamed tissues where the vascular permeability is increased. In an acute mouse colitis model, RANPs effectively scavenged ROS, protected cells from ROS-induced damage, and passively targeted the inflamed colon and protected it from oxidative damage, thereby promoting remission of colonic inflammation without increasing toxicity.

These properties (size and surface charge) are expected to favor particle diffusion, association, and uptake into more-permeable positively charged lesion sites. Although smaller nanoparticles with a modifiable surface can accumulate at the inflammation site through the eEPR effect, ${ }^{70}$ the therapeutic effect of this passive targeting is controversial because of the diversity and complexity of the intestinal microenvironment. PEGylation, a liposome coating, and a negatively charged design of nanoparticles improved the efficiency of passive targeting through the stable transportation of drugs and the eEPR effect. However, DDSs will inevitably enter normal intestinal mucosa.

\section{Active Targeting}

The nanoscale and the surface physicochemical properties of nanoparticles mediating passive accumulation enhance the delivery of drugs to the inflamed area of the colon through stable transportation and the eEPR effect. Although the nanoparticle-based passive target strategy has been shown to be effective in vivo, it still has some limitations. The eEPR effect can only facilitate the accumulation of the DDSs in colitis tissue, and low cellular uptake efficiency by the targeted cells and insufficient intracellular drug release limit the therapeutic efficiency of the anti-inflammatory drugs. Therefore, the development of nanocarriers that can actively target inflammatory cells is expected to achieve more precise spatial positioning to improve the therapeutic effect and minimize adverse effects to the healthy tissues. ${ }^{50}$ For example, CD44, a transmembrane glycoprotein overexpressed by macrophages, is considered to have potential for use in active targeting. ${ }^{71-73}$ In addition, as natural ligands of $\alpha_{4} \beta_{7}$ integrin, ICAM1 ${ }^{74}$ and MAdCAM1 $1^{75}$ have been recognized as markers of IBD, and antiMAdCAM1 and anti-ICAM1 therapies have attracted the attention of clinicians and pharmaceutical companies as promising targets. Therefore, the surface modification 
of nanoparticles with ligands that bind to specific receptor molecules on the membrane can effectively trigger receptor-mediated endocytosis. ${ }^{76,77}$ In this section, some of the active targeting-related research is introduced.

Many receptor-specific ligands can be used to design the oral colon-specific targeting DDSs. For example, hyaluronic acid (HA) with negative charges possesses many advantages, such as nontoxicity, low immunogenicity, and biodegradability, and has been used in ligand-mediated drug delivery. ${ }^{77}$ Generally, HA binds with high affinity to CD44. ${ }^{71}$ Xiao et al designed and produced the HA-functionalized siCD98/CUR-co-loaded polymeric nanoparticles. ${ }^{78}$ The functionalized system performed the targeted drug delivery to the colonic epithelial cells and macrophages by the interaction of HA (ligand) with CD44 (receptor). Overall, polymeric DDSs achieve synergistic treatment through mucosal protection and better therapeutic effects than a single drug. In addition, another similar functionalized system consisting of the HA-KPV-NPs (hyaluronic acid-lysine-proline-valine-nanoparticles) fabricated by the same group showed a similar function in UCrelated cell targeting. ${ }^{79}$

To achieve precise active targeting of drug-loaded nanoparticles and further reduce their side effects, Gou et al designed and produced core-shell nanoparticles composed of the antioxidant and anti-inflammatory drug curcumin (CUR) and the CD44 ligand chondroitin sulfate (CS) (CS-CUR-NPs) through surface modification. ${ }^{80}$ In addition, the CS-CUR-NPs were encapsulated into a hydrogel (chitosan/alginate) to ensure stable transport through the stomach and small intestine and further release of nanoparticles due to their degradation in the targeted area in the inflamed colon. As expected, after oral administration or intravenous (I.V.) injection, the DDS achieved enhanced accumulation through eEPR and regular EPR effects, actively targeting macrophages through the CD44 transcytosis pathway and exhibiting controlled intracellular drug release.

Interestingly, MAdCAM-1 can act as a target in of IBD, and the ablation or inhibition of MAdCAM-1 ameliorates colitis in the DSS-induced IBD. ${ }^{81}$ In another study, Truffi et al developed anti-MAdCAM-1 antibody-functionalized $\mathrm{MnO}$ nanoparticles with low toxicity. ${ }^{82}$ By actively targeting the MAdCAM-1, the $\mathrm{MnO}$ nanoparticles achieved enhanced accumulation in the inflamed bowel sites in a murine model of acute colitis.

Benefiting from good biocompatibility and water insolubility, the $\beta$-glucan-based cytoskeleton of a yeast cell wall with a uniform particle size of $2-4 \mu \mathrm{m}$ acted as a nanocarrier for IBD therapy. ${ }^{83}$ Moreover, $\beta$-glucans are pathogen-associated molecular patterns recognized by dectin-1 and complement receptor 3 (CR3) on the macrophage cell membrane, thus enhancing active targeting-mediated phagocytosis. $^{84}$ Sun et al synthesized yeast cell wall-coated immunosuppressive agent methotrexate (MTX)-loaded nanoparticles (YGPs/MTX) for targeted IBD therapy. ${ }^{85}$ Taken orally, the drug-loaded system preferentially internalized into macrophages, which promoted the uptake of MTX by macrophages, leading to the suppression of NO, TNF- $\alpha$, IL-6, and IL$1 \beta$ secretion. With the active targeting effects, the YGPs/ MTX showed excellent anti-inflammatory effects in vivo.

In addition, the mannose receptor, as a pattern recognition receptor that is exclusively expressed on the surface of macrophages and involved in the immune response, can regulate the activation of macrophages, antigen presentation, and phagocytosis. ${ }^{86}$ Interestingly, studies have shown that mannosylated nanoparticles can precisely target macrophages and not enter systemic circulation. ${ }^{87}$ Sun et al developed mannosylated carbon dot (CD) nanoparticles by covalent conjugation for orally administered IBD therapy. ${ }^{88}$ These CDs/Man-NPs showed excellent accumulation and highly preferred uptake by macrophage cells, indicating their effective active targeting of immunerelated macrophage cells.

As depicted above, macrophage cell surface receptors, such as CD44, dectin-1, CR3 and mannose receptors, showed excellent actively targeted delivery of therapeutic agents for oral administration or intravenous (I.V.) injection. As important antigen-presenting cells in the body, macrophages not only can secrete cytokines (TNF- $\alpha$ ) to regulate the migration and activation of other immune cells but can also participate in the inflammatory response of the intestinal mucosa, thus having the ability to achieve specific actively targeted drug delivery.

\section{Activated/Stimulated Drug Delivery}

Undeniably, passive and active targeting strategies (Figure 3A-C) significantly improved the accumulation efficiency of therapeutic agents in the inflamed colon, which is helpful for effective IBD therapy. Based on the complex physiological and pathological microenvironment of the intestine in vivo, there are many inevitable barriers, making efficient targeted delivery more difficult. For example, after DSS intravenous (I.V.) injection, 
plasma proteins in the blood are the main disruptors of passive targeting because they mask specific ligands. ${ }^{39}$ For administration by injection, DDSs covered in stealth materials to protect guest molecules (small-molecule drugs and biological agents) from destruction and precisely release the drugs at the desired site are urgently needed to improve the therapeutic effects and reduce the toxicity of the DSS. The stealth material is similar to an invisible protective shield and is used to protect guest molecules. After reaching the inflamed colon, as shown in Figure 3D-F, the shield part can be selectively degraded or dissociated by a specific inflammatory intestinal microenvironment ( $\mathrm{pH}, \mathrm{ROS}, \mathrm{GSH}$, enzyme), and the inner surface functional groups of the nanoparticles are re-exposed to promote cell internalization. This "protection/deprotection" targeting strategy activated by the microenvironment is expected to enhance the phagocytic efficiency of the target cells. In this section, the activated/ stimulated responsive delivery strategy is summarized.

\section{$\mathrm{pH}$-Responsive Systems}

As summarized previously, the $\mathrm{pH}$ varies throughout the whole GIT; in contrast to the $\mathrm{pH}$ value in the stomach $(\sim 1.2)$ and proximal small intestine (1.2 and 6.8), the $\mathrm{pH}$ in the inflamed ileum-colon is approximately 7.4. ${ }^{89}$ Because the IBD lesions are concentrated in the ileum and colon, ${ }^{20}$ specific pH-mediated stimulated drug delivery platforms are being developed as options for controlled drug release. Stealth materials such as hydroxypropyl methylcellulose acetate succinate (HPMCAS) polymers, ${ }^{90,91}$ hydrogels ${ }^{92}$ and Eudragit S $100^{93}$ are widely used to design $\mathrm{pH}$-responsive DDSs with core-shell structures, nano-in-micro structures, and hierarchical structures. Some of the solid studies are depicted below.

The stable nanostructure of these materials in an acidic environment makes them efficient for orally administered drug delivery. For instance, acid-resistant HPMCAS is a mixture of acetic acid and monosuccinic acid esters of hydroxypropyl methyl cellulose and can be used as a $\mathrm{pH}-$ responsive shell. ${ }^{94}$ Leveraging this property, Bertoni et al developed intestinal $\mathrm{pH}$-simulated nano-in-micro structured particles (CS-NPs@MF). ${ }^{95}$ After the oral administration, the microparticles $(53 \pm 3 \mu \mathrm{m})$ maintained their integrity in an acidic $\mathrm{pH}$ environment, and then, the protective HPMCAS was selectively degraded in the intestine, close to the targeted site. The tailored system achieved effective protection of the encapsulated nanoparticles and controlled $\mathrm{pH}$-simulated drug release. The results showed decreased drug permeability across the C2BBe1/HT29-MTX cell monolayer and efficient anti-inflammatory effects, thus limiting undesired systemic side effects.

In addition to nano-in-micro structures, core-shell structured pH-responsive DDSs represent one of the many design strategies being investigated for lowering the rate of drug dissolution. ${ }^{96}$ For example, Oshi et al designed and synthesized a hydrogel (chitosan/alginate)based $\mathrm{pH}$-triggered activatable core-shell nanoparticle $\left(\mathrm{CAP}_{1} \mathrm{AG}_{4} \mathrm{CH}_{5} @ \mathrm{CUNC}\right)$ by using ultrasound-assisted antisolvent crystallization and LBL-coating techniques for use in drug delivery for IBD therapy. ${ }^{92}$ The coreshell structure system is composed of an inner curcumin nanocrystals (CUNC) core surrounded by chitosan $(\mathrm{CH})$, sodium alginate $(\mathrm{AG})$, and cellulose acetate phthalate

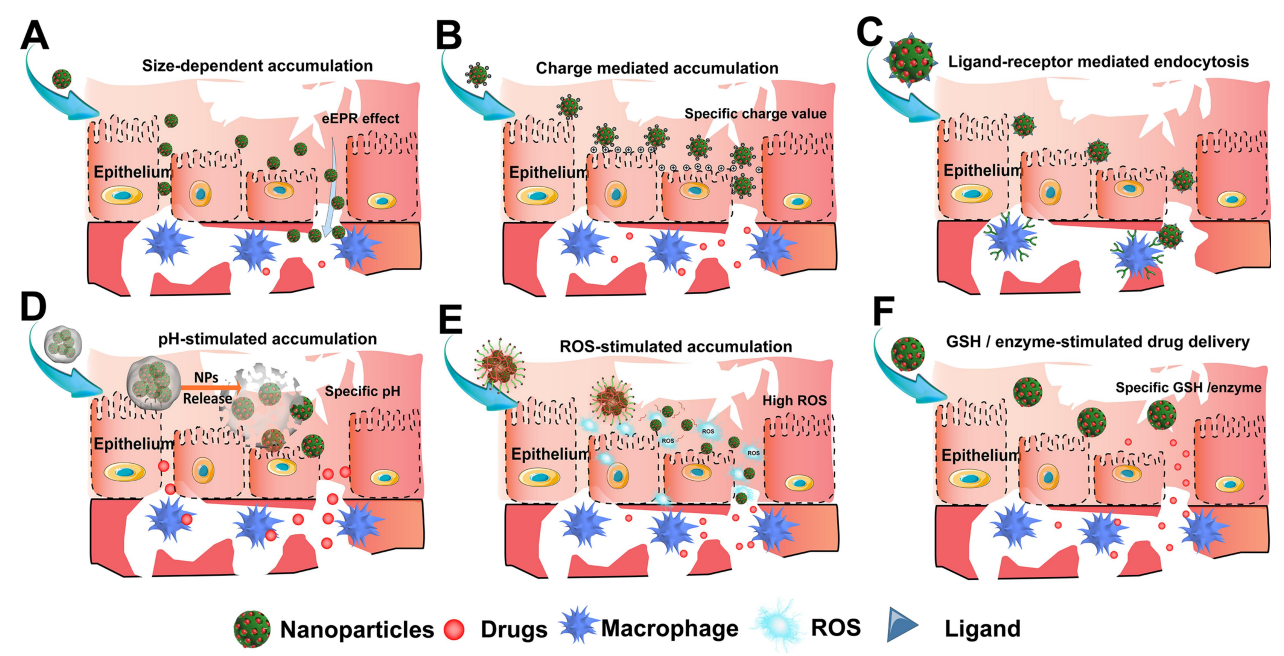

Figure 3 Schematic illustration of the scheme of inflammation-targeting in IBD. Through oral administration and rectal administration (I.V. administration are not depicted here), nanoparticles target the inflamed colon by mechanisms mediated by size (A), charge (B), ligand-receptor (C), pH (D), ROS (E), GSH and enzyme (F). 
(CAP). Due to the insoluble properties of CAP in the gastric $\mathrm{pH}$, the system remained intact in the upper GIT. Upon reaching the colon, CAP was dissolved and underwent charge inversion on the particle surface and then achieved enhanced accumulation in inflamed colon through the eEPR effect. This $\mathrm{pH}$-mediated charge-reversing nanoplatform can not only prevent drug leakage but also deliver precise IBD therapy.

Eudragit is an anionic copolymer of methacrylic acid and methyl methacrylate, Eudragit $\mathrm{S}$ is soluble at $\mathrm{pH}>7$ and usually used for colon targeting. In recent years, a large number of Eudragit-based colon-targeting DDSs have been developed. In a classic study, Oshi and coworkers developed $\mathrm{pH}$-responsive colon-targeted Eudragit S 100 (ES)-coated nanoparticles (ES1AG4CH5-DXMCs) using a layer-by-layer coating technique. ${ }^{93}$ The DDS prevented the premature drug release by the ES layer, and then, the dissolution of the ES layer led to sustained drug release in the colon. Moreover, ideal colon-specific, dualfunctional CSA-loaded polymeric nanoparticles produced by the combination of a PLGA copolymer and pH-responsive Eudragit FS30D polymer (Eudragit FS30D/PLGA nanoparticles) for use in UC therapy were prepared.$^{97}$ These DDSs showed incomplete and slow release of $\mathrm{CSA}$ at the $\mathrm{pH}$ of the ileum and colon, followed by sustained release, thus delivering a sufficient amount of CSA specifically to the inflamed colon. In vivo experiments showed that these nanoparticles had a significant effect in alleviating colitis and appeared to be a promising safe and effective drug delivery system for UC therapy. Overall, pH-mediated DDSs can be used in an efficient strategy for targeted drug delivery in IBD therapy.

\section{Endogenous ROS-Responsive Systems}

Studies have shown that reactive oxygen species (ROS) produced by activated macrophages and leukocytes in the area of colitis, activating inflammatory response pathways (MMPs, (TLR4)-NF- $\mathrm{kB}, \mathrm{NLRP} 3$ ), ${ }^{98}$ and oxidative damage to DNA, proteins, and lipids caused by the local overproduction of ROS may promote the initiation and progression of UC..$^{99}$ ROS are highly reactive ions and free radicals, including superoxide $\left(\mathrm{O}^{2-}\right)$, hydroxyl radicals $(\cdot \mathrm{OH})$, hypochlorite ions $\left(\mathrm{OCl}^{-}\right)$, hydrogen peroxide $\left(\mathrm{H}_{2} \mathrm{O}_{2}\right)$, and singlet oxygen $\left({ }^{1} \mathrm{O}_{2}\right) .{ }^{100}$ Targeting the sites of inflammation and scavenging ROS may constitute an efficient strategy to attenuate colitis. In recent years, to stabilize the microenvironment in pathological tissue, nanoparticle regulation of ROS levels in the inflamed region has been widely studied. ${ }^{101}$ With the development and research of nanoparticles that are suitable for the GI drug delivery, ROS not only can serve as targets at inflammation sites but can also be used as signals to trigger responsive micro/nanomaterials. ${ }^{102}$

Considering the high oxidative stress characteristics of an inflammatory site, ROS-responsive micro-and nanodrug carriers have been widely studied to stabilize the microenvironment in the pathological tissue because of their excellent drug transportability and tissue permeability, leading to new antioxidant therapies for IBD therapy. ${ }^{102}$ Multifunctional DDSs based on ROS-responsive groups, such as poly (propylene sulfide) and thioketal linkers, ${ }^{102,103}$ are highly sensitive to $\mathrm{H}_{2} \mathrm{O}_{2} .{ }^{104,105}$

Due to the unfavorable microenvironment of the GIT and the specific high oxidative stress of the inflammatory site, $\mathrm{Li}$ et $\mathrm{al}^{106}$ developed ROS-responsive material (OxbCD)-based NPs for Ac2-26 peptide delivery of orally administered therapeutic drugs for IBD relief research. Smart nanocarriers were constructed with $\beta$-cyclodextrin ( $\beta$-CD) and 4-(hydroxymethyl) phenylboronic acid pinacol ester (PBAP). The drug-loading system can maintain its stability and the bioactivity of the drug in the normal gastrointestinal tract. Due to the decrease in the thickness of the mucus layer and the destruction of the intestinal barrier in IBD, the drug delivery system can enrich specific inflammatory sites. In addition, the structure can be changed under the stimulation of ROS at high levels in the inflamed site, which leads to drug release, and thereby effective inflammation relief can be attained. In vitro and in vivo experiments showed an enhanced efficacy of Ac226 through the protective transport of peptides and precise ROS-responsive delivery to inflammatory sites. In addition, this nanocarrier can effectively inhibit the apoptosis of macrophages, reduce the levels of ROS, and enhance the clearance of apoptotic neutrophils by macrophages.

In addition, the same research group designed a selfassembled and oxidizable Janus prodrug, Bud-ATK-Tem (B-ATK-T). ${ }^{101}$ This drug-carrier system was prepared with budesonide (41.23\%) and tempol (15.55\%) as the main therapeutic drugs, which were linked by ATK, and it caused few side effects. Strikingly, this study confirmed that orally administered B-ATK-T-NPs can passively target colitis sites and release Bud and Tem upon triggering by ROS in the colon tissue to prevent potential systemic side effects.

ROS-responsive oral drug carriers have also been used in colorectal cancer by Zhang et al. ${ }^{107}$ Studies pertinent to inflammation and cancer have demonstrated that the long- 
term oxidative stress caused by the excessive production of ROS promoted inflammation and cancer to a great extent. $^{108}$ Zhang et al synthesized a ROS-responsive, hydrogen peroxide-scavenging material loaded with camptothecin-11 (CPT-11). This drug carrier was manufactured by chemical functionalization of $\beta-C D$ with an oxidationlabile moiety of AM-PBAP. In vitro drug release experiments showed a higher and sustained profile upon high oxidative stress stimulation and intestinal inflammation $\mathrm{pH}$ conditions. Moreover, in vivo, oral delivery of this drug delivery system significantly inhibited the tumorigenesis and growth of induced CAC in mice and showed good therapeutic effects.

In general, ROS-responsive biomaterials have been developed and used in the study of oral medication for IBD and CAC, especially ROS-responsive thioketal nanoparticles. ${ }^{109,110}$ Therefore, ROS-sensitive bonds also act as ROS scavengers to reduce oxidative stress. However, although most show no cytotoxicity, these biomaterials should be made to be highly biocompatible, and fragments of the material degraded in vivo should not trigger further inflammatory reactions.

\section{GSH-Responsive Systems}

Based on previous studies, although the extracellular environment is oxidizing, the intracellular compartment is in a reductive condition, and the amount of intracellular soluble GSH is significantly elevated during the activation of macrophages. ${ }^{111}$ The special microenvironment created by GSH makes it a suitable stimulus signal for targeted drug delivery. ${ }^{112}$ Therefore, this strategy combined with the eEPR effect to achieve enhanced accumulation and targeted drug release is expected to achieve precise therapy of tumors.

Xiao and coworkers loaded polyphenol epigallocatechin gallate (EGCG) into the globular glycoprotein ovalbumin (OVA) via self-assembly. ${ }^{113}$ Nanoparticles ( 202.9 $\mathrm{nm}$ ) were thus obtained with a negative surface charge and displayed enhanced accumulation and glutathione-responsive drug release behavior in a reductive microenvironment. The in vivo experiments clearly indicated that intravenously administered EGCG-NPs produced significantly better therapeutic outcomes against UC than pristine EGCG.

\section{Enzyme-Responsive Systems}

Among the aforementioned systems, microbiota-activated DDSs have been developed for IBD therapy, as they exert their therapeutic effects in the presence of specific enzymes (azoreductase, $\beta$-galactosidase, $\beta$-xylosidase, nitroreductase, glycosidase, and deaminase) produced by the colonic bacteria. ${ }^{114}$ These enzymes, such as azoreductase produced by the gut microbiota for the degradation of azobenzene (Azo), were studied by Cheng et al. ${ }^{115}$ Based on the presence of azoreductase, which is specifically produced by the gut microbiota and is abundant in the colon tract, the enzyme-mediated platform (Trp·Azo) $\subset \mathrm{CB}[8]$ was developed by assembling chitosan/hyaluronic acid (CS/HA) as a "gate-keepers" molecule on the periphery of a mesoporous silica core. After oral administration, the formed DDS was disassembled in the presence of azoreductase, and the inner parts were re-exposed to the inflamed area. In addition, Trp derivatives attached to the polymer backbone of CS are converted to various Trp metabolites by the gut microbiota, functioning as AHR agonists.

In summary, the goal of IBD therapy is that therapeutic agents exert only the effects in the inflamed areas, while they are inactivated in healthy tissue and blood circulation, which will significantly improve the efficacy of IBD therapy. The unique GIT microenvironment of $\mathrm{pH}, \mathrm{ROS}, \mathrm{GSH}$ and enzymes can be used as stimulus signals. Stimulated drug delivery strategies based on the internal GIT microenvironment are summarized in Table 1. Studies showed that activatable strategies can minimize the nonselective harmful effects on normal tissue, which is helpful to broaden the clinical application of nanoparticle-based IBD therapy.

\section{Nanocarriers Mediate Precise Diagnoses of IBD}

Assessment of IBD currently relies on specific clinical signs of bowel inflammation. With the advancement of cross-sectional imaging techniques such as computed tomography (CT) copy, radiologic techniques play integral roles in the diagnosis and management of patients with IBD. Imaging has long been utilized to evaluate segments of the digestive tract that are inaccessible with conventional endoscopy. For decades, nanoparticles such as $\mathrm{Au},{ }^{116,117} \mathrm{Fe}$ and $\mathrm{Au}$ complexes, ${ }^{118}$ quantum dots, ${ }^{119,120}$ $\mathrm{MnO},{ }^{82}$ and lipid nanoparticles ${ }^{74}$ have been developed and used in cancer imaging and diagnosis. During traditional clinical CT imaging, iodinated small molecules and barium sulfate suspensions are usually used as contrast agents, ${ }^{121,122}$ but they are not ideal for imaging IBD due to their nonspecificity. Furthermore, when administered 
Table I Recent Representative Advances of Inflamed Intestinal Microenvironment-Stimulated Drug Delivery Platforms for IBD Therapy

\begin{tabular}{|c|c|c|c|c|}
\hline System & Size & Model Drug & Target Mechanism & Ref \\
\hline AP@PSi-HA@HPMCAS & $35 \pm 1 \mu \mathrm{m}$ & $\begin{array}{l}\text { Budesonide (BUD) and acorbyl } \\
\text { palmitate (AP) }\end{array}$ & $\mathrm{pH}$-stimulated & {$[62]$} \\
\hline YGPs/MTX & $2-4 \mu \mathrm{m}$ & Methotrexate & $\mathrm{pH}$-stimulated & {$[85]$} \\
\hline HNT-ATV@HF-CEL & $70 \pm 8 \mu \mathrm{m}$ & Atorvastatin and celecoxib & $\mathrm{pH}$-stimulated & {$[90]$} \\
\hline $\mathrm{CAP}_{1} \mathrm{AG}_{4} \mathrm{CH}_{5} @ \mathrm{CUNCs}$ & $421 \pm 14 \mathrm{~nm}$ & Curcumin & $\mathrm{pH}$-stimulated & [92] \\
\hline OxiDEX nano-in-micro composites & $53 \pm 3 \mu \mathrm{m}$ & Rifaximin & $\begin{array}{l}\mathrm{pH} \text { and ROS-Sequential } \\
\text { Responsive }\end{array}$ & [95] \\
\hline P(LE-IAMEG) hydrogel microspheres (HMSs) & $34.87 \pm 0.90 \mu \mathrm{m}$ & $\begin{array}{l}\text { Hydrocortisone sodium succinate } \\
\text { (HSS) }\end{array}$ & $\mathrm{pH}$-stimulated & [128] \\
\hline BLG-RES nanospheres & $200-250 \mathrm{~nm}$ & Resveratrol & $\mathrm{pH}$-stimulated & [129] \\
\hline OxbCD & $202 \pm 4 \mathrm{~nm}$ & $\begin{array}{l}\text { Annexin Al-mimetic peptide Ac2- } \\
26\end{array}$ & ROS-stimulated & [106] \\
\hline Bud-ATK-Tem (B-ATK-T) & $100 \sim 120 \mathrm{~nm}$ & Budesonide and antioxidant tempol & ROS-stimulated & [10I] \\
\hline RANPs & $63.5 \pm 4.0 \mathrm{~nm}$ & Dexamethasone & ROS-stimulated & [67] \\
\hline EGCG-NPs & $\sim 202.9 \mathrm{~nm}$ & Epigallo-catechin 3-gallate & GSH-stimulated & [II3] \\
\hline MSs (multilayer-coated mesoporous silica) & $210-258 \mathrm{~nm}$ & Hydrocortisone & Enzyme-stimulated & [115] \\
\hline $\begin{array}{l}\text { PPNP (polyphenols and polymers self- } \\
\text { assembled nanoparticle) }\end{array}$ & $\sim 60 \mathrm{~nm}$ & Dexamethasone & Enzyme-stimulated & [130] \\
\hline
\end{tabular}

orally, these suspensions are nonspecifically distributed throughout the GIT, thus interfering with diagnosis and leading to accumulative radiation exposure in IBD patients. $^{123}$ Therefore, the CT contrast agent must not only be stable in the GIT microenvironment but also must be specifically enriched in the diseased parts of the intestine to better detect disease.

Based on the microenvironmental characteristics of the GIT and the design strategy of orally administered DDSs for IBD therapy, the application of nanoparticles may create ideas for the CT imaging in IBD. Naha et al designed and synthesized nanocontrast agents that can be used in the diagnosis and treatment of IBD, which were named dextran-coated cerium oxide nanoparticles (DexCeNPs). ${ }^{124}$ This nanocontrast agent consists of a cerium oxide core and a dextran shell, which can endow the system with strong CT contrast due to the higher k-edge of cerium oxide and the stability, biocompatibility, and specificity in the inflammation sites due to the unique physicochemical properties of dextran. ${ }^{125,126}$ Furthermore, cerium oxide can act as an artificial ROS scavenger ${ }^{127}$ to reduce the high oxidative stress in intestinal lesions to alleviate inflammation. As demonstrated with a clinical CT scanner, in healthy mice and DSSinduced colitis mice, Dex-CeNPs produced a strong CT contrast in the GIT and accumulated in the tissues affected by colitis. ICP-OES and TEM confirmed the accumulation of Dex-CeNPs in the large intestine of mice with colitis $24 \mathrm{~h}$ post-administration. Notably, $99.9 \%$ and $97.6 \%$ of the oral dose was cleared from the body of healthy mice and mice with colitis within $24 \mathrm{~h}$, respectively.

In another study, Fabio Corsi and coworkers loaded quantum dots into anti-MAdCAM-1 coupled PLGAPEG nanoparticles (P@QD-MdC NPs). ${ }^{120}$ Upon intravenous injection, the system is expected to specifically bind to MAdCAM-1 protein on the apical side of mucosal endothelial vessels and penetrate the bowel mucosa. 
In vivo experiments showed progressive P@QD-MdC NP accumulation in the inflamed colon and provided an enhanced and localized signal.

Overall, nanoparticles not only can be designed and produced as drug carriers for the treatment of IBD but can also be potential CT contrast agents for GIT imaging, including IBD imaging. Therefore, they will definitely play greater roles in future IBD diagnostic applications.

\section{Conclusions, Challenges, and Perspectives}

DDSs with drug-loading capacity bring hope for IBD therapy and diagnosis. Their unique advantages, such as facile surface modification, make it possible for nanoparticles to engage in passive targeting, active targeting, and stimulated targeting-mediated delivery of therapeutic agents. Targeting drug delivery aims to ensure direct treatment at the inflammatory site, avoid the pre-metabolism of the system, and reduce drug absorption in the small intestine and other nonspecific areas to improve the therapeutic effect and reduce side effects. Besides, the emerging researches in cell biology and microbiology have spotlighted the increasing importance of cell-and fecal microbiota transplantation based IBD therapy, which offers the potential of altering and treating the course of diseases that cannot be addressed sufficiently by existing pharmaceuticals. With the increasing development of nanomedicine, nanoparticles based microbiota therapy will be a hotspot, such as surface decoration of living cells to protect its bioactivity. Nevertheless, there will be a long period from nanodrugs to clinical translation and increasing efforts and research should be performed to hasten DDS application. Interestingly, to solve these potential problems, researchers should develop a deep understanding of the interaction between nanomaterials and IBD and the potential for the clinical application of nanomaterials in diagnosis and treatment. Moreover, targeting multifunctional materials integrating diagnosis and treatment will be the future direction. Further, efforts should be made to evaluate their toxicity in the long term and formulate relevant regulatory programs. With these strenuous efforts, it is hoped that this exciting technology can provide effective and personalized treatment and diagnosis to the management of IBD.

\section{Abbreviations}

IBD, inflammatory bowel disease; MDR, multidrug resistance; GIT, gastrointestinal tract; DDSs, drug delivery systems; eEPR, enhanced epithelial permeability and retention; ROS, reactive oxygen species.

\section{Acknowledgments}

This research was supported by grants from the National Natural Science Foundation of China (NSFC 81873551 to H.Y. and NSFC 81970468 to M.Y.), and Innovative Research Team of Ministry of Education of China (IRT_17R16 to H.Y.).

\section{Disclosure}

The authors report no conflicts of interest in this work.

\section{References}

1. Kaser A, Zeissig S, Blumberg RS. Inflammatory bowel disease. Anпu Rev Immunol. 2010;28(1):573-621. doi:10.1146/annurevimmunol-030409-101225

2. Alhouayek M, Muccioli GG. The endocannabinoid system in inflammatory bowel diseases: from pathophysiology to therapeutic opportunity. Trends Mol Med. 2012;28:615-625. doi:10.1016/ j.molmed.2012.07.009

3. Moran CJ, Huang H, Rivas M, et al. Genetic variants in cellular transport do not affect mesalamine response in ulcerative colitis. PLoS One. 2018;13(3):e0192806. doi:10.1371/journal. pone.0192806

4. Taylor KM, Irving PM. Optimization of conventional therapy in patients with IBD. Nat Rev Gastroenterol Hepatol. 2011;8 (11):646. doi:10.1038/nrgastro.2011.172

5. Bryant RV, Brain O, Travis SPL. Conventional drug therapy for inflammatory bowel disease. Scand J Gastroenterol. 2015;50(1)::90-112. doi:10.3109/00365521.2014.968864

6. Cario E. P-glycoprotein multidrug transporter in inflammatory bowel diseases: more questions than answers. World J Gastroenterol. 2017;23:1513-1520.

7. Walker G, Ahmad T. Drug toxicity: personalising IBD therapeutics - the use of genetic biomarkers to reduce drug toxicity. In: Sheng Ding N, De Cruz P, editors. Biomarkers in Inflammatory Bowel Diseases. Cham: Springer International Publishing; 2019:257-269.

8. Nance E, Zhang C, Shih T-Y, et al. Brain-penetrating nanoparticles improve paclitaxel efficacy in malignant glioma following local administration. ACS Nano. 2014;8:10655-10664.

9. Lamprecht A. Selective nanoparticle adhesion can enhance colitis therapy. Nat Rev Gastroenterol Hepatol. 2010;7:311.

10. Hua S, Marks E, Schneider JJ, et al. Advances in oral nano-delivery systems for colon targeted drug delivery in inflammatory bowel disease: selective targeting to diseased versus healthy tissue. Nanomedicine. 2015;11(5):1117-1132. doi:10.1016/j.nano.2015.02.018

11. Mittal R, Patel AP, Jhaveri VM, et al. Recent advancements in nanoparticle based drug delivery for gastrointestinal disorders. Expert Opin Drug Deliv. 2018;15(3):301-318. doi:10.1080/ 17425247.2018.1420055

12. Sahoo SK, Misra R, Parveen S. Nanoparticles: a boon to drug delivery, therapeutics, diagnostics and imaging. In: Nanomedicine in Cancer. Pan Stanford; 2017:73-124. 
13. Guada M, Beloqui A, Alhouayek M, et al. Cyclosporine A-loaded lipid nanoparticles in inflammatory bowel disease. Int J Pharm. 2016;503(1-2):196-198. doi:10.1016/j.ijpharm.2016.03.012

14. Mir M, Ahmed N, Rehman AU. Recent applications of PLGA based nanostructures in drug delivery. Colloids Surf B Biointerfaces. 2017;159:217-231. doi:10.1016/j.colsurfb.2017.07.038

15. Hossain MA, Yamashita M, Vong LB, et al. Silica-installed redox nanoparticles for novel oral nanotherapeutics - improvement in intestinal delivery with anti-inflammatory effects. J Drug Target. 2014;22(7):638-647. doi:10.3109/1061186X.2014.928716

16. Yang B, Jiang J, Jiang L, et al. Chitosan mediated solid lipid nanoparticles for enhanced liver delivery of zedoary turmeric oil in vivo. Int J Biol Macromol. 2020;149:108-115. doi:10.1016/j. ijbiomac.2020.01.222

17. Zhai J, Mantaj J, Vllasaliu D. Ascorbyl palmitate hydrogel for local, intestinal delivery of macromolecules. Pharmaceutics. 2018;10(4):188. doi:10.3390/pharmaceutics 10040188

18. Tong T, Qi Y, Bussiere LD, et al. Transport of artificial virus-like nanocarriers through intestinal monolayers via microfold cells. Nanoscale. 2020;12(30):16339-16347. doi:10.1039/ D0NR03680C

19. Argyo C, Weiss V, Bräuchle C, et al. ChemInform abstract: multifunctional mesoporous silica nanoparticles as a universal platform for drug delivery. Cheminform. 2014;45(11):435-451. doi:10.1002/chin.201411272

20. Youshia J, Lamprecht A. Size-dependent nanoparticulate drug delivery in inflammatory bowel diseases. Expert Opin Drug Deliv. 2016;13(2):281-294. doi:10.1517/17425247.2016.1114604

21. Yong MJ, Mantaj J, Cheng Y, et al. Delivery of nanoparticles across the intestinal epithelium via the transferrin transport pathway. Pharmaceutics. 2019;11(7):298. doi:10.3390/ pharmaceutics11070298

22. Bharti C, Nagaich U, Pal AK, et al. Mesoporous silica nanoparticles in target drug delivery system: a review. Int J Pharm Investig. 2015;5:124-133.

23. Beloqui A, Coco R, Memvanga PB, et al. pH-sensitive nanoparticles for colonic delivery of curcumin in inflammatory bowel disease. Int J Pharm. 2014;473:203-212.

24. Yao Y, Zhang $H$, Wang $Z$, et al. Reactive oxygen species (ROS)-responsive biomaterials mediate tissue microenvironments and tissue regeneration. J Mater Chem B. 2019;7(33):5019-5037. doi:10.1039/C9TB00847K

25. Xu W, Ling $\mathrm{P}$, Zhang T. Polymeric micelles, a promising drug delivery system to enhance bioavailability of poorly water-soluble drugs Journal of Drug Delivery. 2013;2013:15.

26. Felber AE, Dufresne M-H, Leroux J-C. pH-sensitive vesicles, polymeric micelles, and nanospheres prepared with polycarboxylates. Adv Drug Del Rev. 2012;64:979-992. doi:10.1016/j.addr.2011.09.006

27. Sood A, Panchagnula R. Peroral route: an opportunity for protein and peptide drug delivery. Chem Rev. 2001;101(11):3275-3304 doi:10.1021/cr000700m

28. Bertoni S, Machness A, Tiboni M, et al. Reactive oxygen species responsive nanoplatforms as smart drug delivery systems for gastrointestinal tract targeting. Biopolymers. 2020;111(1): e23336. doi:10.1002/bip.23336

29. Sogias IA, Williams AC, Khutoryanskiy VV. Why is chitosan mucoadhesive? Biomacromolecules. 2008;9(7):1837-1842. doi:10.1021/bm800276d

30. Cook MT, Tzortzis G, Charalampopoulos D, et al. Microencapsulation of probiotics for gastrointestinal delivery. J Control Release. 2012;162(1):56-67. doi:10.1016/j. jconrel.2012.06.003

31. Khutoryanskiy VV. Advances in mucoadhesion and mucoadhesive polymers. Macromol Biosci. 2011;11(6):748-764. doi:10.1002/mabi.201000388
32. Sartor RB. Microbial influences in inflammatory bowel diseases Gastroenterology. 2008;134(2):577-594. doi:10.1053/j. gastro.2007.11.059

33. Alam MT, Amos GCA, Murphy ARJ, et al. Microbial imbalance in inflammatory bowel disease patients at different taxonomic levels. Gut Pathog. 2020;12(1):1. doi:10.1186/s13099-019-0341-6

34. Musch MW, Wang Y, Claud EC, et al. Lubiprostone decreases mouse colonic inner mucus layer thickness and alters intestinal microbiota. Dig Dis Sci. 2013;58(3):668-677. doi:10.1007/ s10620-012-2509-5

35. Johnstone RW, Ruefli AA, Smyth MJ. Multiple physiological functions for multidrug transporter P-glycoprotein? Trends Biochem Sci. 2000;25(1):1-6. doi:10.1016/S0968-0004(99)01493-0

36. Chan LMS, Lowes S, Hirst BH. The ABCs of drug transport in intestine and liver: efflux proteins limiting drug absorption and bioavailability. Eur J Pharm Sci. 2004;21(1):25-51. doi:10.1016/ j.ejps.2003.07.003

37. Gavhane YN, Yadav AV. Loss of orally administered drugs in GI tract. Saudi Pharm J. 2012;20(4):331-344. doi:10.1016/j. jsps.2012.03.005

38. Takano M, Yumoto R, Murakami T. Expression and function of efflux drug transporters in the intestine. Pharmacol Ther. 2006;109(1-2):137-161. doi:10.1016/j.pharmthera.2005.06.005

39. Liu J, Li M, Luo Z, et al. Design of nanocarriers based on complex biological barriers in vivo for tumor therapy. Nano Today. 2017;15:56-90. doi:10.1016/j.nantod.2017.06.010

40. Klotz U, Schwab M. Topical delivery of therapeutic agents in the treatment of inflammatory bowel disease. Adv Drug Del Rev. 2005;57(2):267-279. doi:10.1016/j.addr.2004.08.007

41. Frei P, Biedermann L, Manser $\mathrm{CN}$, et al. Topical therapies in inflammatory bowel disease. Digestion. 2012;86(s1):36-44. doi:10.1159/000341947

42. Mariangela A, Gionata F, Daniela G, et al. Biologic therapies in ulcerative colitis: primi inter pares? Curr Drug Targets. 2018;19 (7):748-756. doi:10.2174/1389450117666160527142719

43. Fiorino G, Fazio M, Danese S. Biosimilar therapies in inflammatory bowel disease: should we care about patient profile? Expert Rev Clin Immunol. 2016;12(4):361-363. doi:10.1586/ 1744666X.2016.1141050

44. Iqbal S, Du X, Wang J, et al. Surface charge tunable nanoparticles for TNF- $\alpha$ siRNA oral delivery for treating ulcerative colitis. Nano Res. 2018;11(5):2872-2884. doi:10.1007/s12274-0171918-3

45. Goldberg M, Gomez-Orellana I. Challenges for the oral delivery of macromolecules. Nat Rev Drug Discov. 2003;2(4):289-295. doi: $10.1038 /$ nrd1067

46. Anselmo AC, Gokarn Y, Mitragotri S. Non-invasive delivery strategies for biologics. Nat Rev Drug Discov. 2019;18 (1):19-40. doi:10.1038/nrd.2018.183

47. Chung SW, Hil-lal TA, Byun Y. Strategies for non-invasive delivery of biologics. J Drug Target. 2012;20(6):481-501. doi:10.3109/1061186X.2012.693499

48. Ram PD, Vishwa VG, Beena GS, et al. Passive and active drug targeting: role of nanocarriers in rational design of anticancer formulations. Curr Pharm Des. 2019;25(28):3034-3056. doi:10.2174/1381612825666190830155319

49. Alavi M, Hamidi M. Passive and active targeting in cancer therapy by liposomes and lipid nanoparticles. Drug Metab Pers Ther. 2019;34(1). doi:10.1515/dmpt-2018-0032

50. Zhang X, Wu W. Ligand-mediated active targeting for enhanced oral absorption. Drug Discov Today. 2014;19(7):898-904. doi:10.1016/j.drudis.2014.03.001

51. Yun Y, Cho YW, Park K. Nanoparticles for oral delivery: targeted nanoparticles with peptidic ligands for oral protein delivery. $A d v$ Drug Del Rev. 2013;65(6):822-832. doi:10.1016/j. addr.2012.10.007 
52. Herrero EP, Alonso MJ, Csaba N. Polymer-based oral peptide nanomedicines. Ther Deliv. 2012;3(5):657-668. doi:10.4155/ tde. 12.40

53. Lu L, Chen G, Qiu Y, et al. Nanoparticle-based oral delivery systems for colon targeting: principles and design strategies. Sci Bull. 2016;61:670-681.

54. Schmidt C, Lautenschlaeger C, Collnot E-M, et al. Nano- and microscaled particles for drug targeting to inflamed intestinal mucosa-a first in vivo study in human patients. $J$ Control Release. 2013;165:139-145.

55. Abdelmegid AM, Abdo FK, Ahmed FE, et al. Therapeutic effect of gold nanoparticles on DSS-induced ulcerative colitis in mice with reference to interleukin-17 expression. Sci Rep. 2019;9:10176.

56. Kim JJ, Bridle BW, Ghia J-E, et al. Targeted inhibition of serotonin type $7\left(5-\mathrm{HT}_{7}\right)$ receptor function modulates immune responses and reduces the severity of intestinal inflammation. J Immunol. 2013;190:4795.

57. Tirosh B, Khatib N, Barenholz Y, et al. Transferrin as a luminal target for negatively charged liposomes in the inflamed colonic mucosa. Mol Pharm. 2009;6:1083-1091.

58. Peterson CGB, Eklund E, Taha Y, et al. A new method for the quantification of neutrophil and eosinophil cationic proteins in feces: establishment of normal levels and clinical application in patients with inflammatory bowel disease. Am J Gastroenterol. 2002;97:1755-1762.

59. Canny G, Levy O, Furuta GT, et al. Lipid mediator-induced expression of bactericidal/permeability-increasing protein (BPI) in human mucosal epithelia. Proc Natl Acad Sci $U S A$. 2002;99:3902-3907.

60. Courthion H, Mugnier T, Rousseaux C, et al. Self-assembling polymeric nanocarriers to target inflammatory lesions in ulcerative colitis. J Control Release. 2018;275:32-39.

61. Lee Y, Sugihara K, Gillilland MG, et al. Hyaluronic acid-bilirubin nanomedicine for targeted modulation of dysregulated intestinal barrier, microbiome and immune responses in colitis. Nat Mater. 2020;19:118-126.

62. Li W, Li Y, Liu Z, et al. Hierarchical structured and programmed vehicles deliver drugs locally to inflamed sites of intestine. Biomaterials. 2018;185:322-332.

63. Zhao J, Gao W, Cai X, et al. Nanozyme-mediated catalytic nanotherapy for inflammatory bowel disease. Theranostics. 2019;9:2843-2855.

64. Zhang S, Cho WJ, Jin AT, et al. Heparin-coated albumin nanoparticles for drug combination in targeting inflamed intestine. $A d v$ Healthcare Mater. 2020;9:2000536.

65. Zhang L, Gui S, Xu Y, et al. Colon tissue-accumulating mesoporous carbon nanoparticles loaded with Musca domestica cecropin for ulcerative colitis therapy. Theranostics. 2021;11:3417-3438

66. Zhao S, Li Y, Liu Q, et al. An orally administered CeO2@montmorillonite nanozyme targets inflammation for inflammatory bowel disease therapy. Adv Funct Mater. 2020;30:2004692.

67. Chung $\mathrm{CH}$, Jung W, Keum H, et al. Nanoparticles derived from the natural antioxidant rosmarinic acid ameliorate acute inflammatory bowel disease. ACS Nano. 2020;14:6887-6896.

68. Lee Y, Lee S, Jon S. Biotinylated bilirubin nanoparticles as a tumor microenvironment-responsive drug delivery system for targeted cancer therapy. Adv Sci. 2018;5:1800017.

69. Lee S, Lee Y, Kim H, et al. Bilirubin nanoparticle-assisted delivery of a small molecule-drug conjugate for targeted cancer therapy. Biomacromolecules. 2018;19:2270-2277.

70. Xiao B, Merlin D. Oral colon-specific therapeutic approaches toward treatment of inflammatory bowel disease. Expert Opin Drug Deliv. 2012;9:1393-1407.
71. Dreaden EC, Morton SW, Shopsowitz KE, et al. Bimodal tumor-targeting from microenvironment responsive hyaluronan layer-by-layer (LbL) nanoparticles. ACS Nano. 2014;8:8374-8382.

72. Rios de la Rosa JM, Tirella A, Gennari A, et al. The CD44-mediated uptake of hyaluronic acid-based carriers in macrophages. Adv Healthcare Mater. 2017;6:1601012.

73. Na YR, Stakenborg M, Seok SH, et al. Macrophages in intestinal inflammation and resolution: a potential therapeutic target in IBD. Nat Rev Gastroenterol Hepatol. 2019;16:531-543.

74. Tlaxca JL, Rychak JJ, Ernst PB, et al. Ultrasound-based molecular imaging and specific gene delivery to mesenteric vasculature by endothelial adhesion molecule targeted microbubbles in a mouse model of Crohn's disease. J Control Release. 2013;165:216-225.

75. Bachmann C, Klibanov AL, Olson TS, et al. Targeting Mucosal Addressin Cellular Adhesion Molecule (MAdCAM)-1 to noninvasively image experimental Crohn's disease. Gastroenterology. 2006;130:8-16.

76. Zhang M, Yang C, Merlin D. P084 oral delivery of nanoparticles loaded with ginger active compound, 6-shogaol, attenuates ulcerative colitis and promotes wound healing in a murine model of ulcerative colitis. Gastroenterology. 2018;154:S44.

77. Zhang M, Xu C, Wen L, et al. A hyaluronidase-responsive nanoparticle-based drug delivery system for targeting colon cancer cells. Cancer Res. 2016;76:7208.

78. Xiao B, Zhang Z, Viennois E, et al. Combination therapy for ulcerative colitis: orally targeted nanoparticles prevent mucosal damage and relieve inflammation. Theranostics. 2016;6:2250-2266.

79. Xiao $\mathrm{B}, \mathrm{Xu} \mathrm{Z}$, Viennois E, et al. Orally targeted delivery of tripeptide KPV via hyaluronic acid-functionalized nanoparticles efficiently alleviates ulcerative colitis. Mol Ther. 2017;25:1628-1640.

80. Gou S, Huang Y, Wan Y, et al. Multi-bioresponsive silk fibroin-based nanoparticles with on-demand cytoplasmic drug release capacity for CD44-targeted alleviation of ulcerative colitis. Biomaterials. 2019;212:39-54.

81. Schippers A, Muschaweck M, Clahsen T, et al. $\beta 7$-Integrin exacerbates experimental DSS-induced colitis in mice by directing inflammatory monocytes into the colon. Mucosal Immunol. 2016;9:527-538.

82. Truffi $\mathrm{M}$, Colombo M, Peñaranda-Avila J, et al. Nano-targeting of mucosal addressin cell adhesion molecule-1 identifies bowel inflammation foci in murine model. Nanomedicine. 2017;12:1547-1560.

83. Ooi VE, Liu F. Immunomodulation and anti-cancer activity of polysaccharide-protein complexes. Curr Med Chem. 2000;7:715-729.

84. Chan GC-F, Chan WK, Sze DM-Y. The effects of $\beta$-glucan on human immune and cancer cells. J Hematol Oncol. 2009;2:25.

85. Sun Y, Duan B, Chen H, et al. A novel strategy for treating inflammatory bowel disease by targeting delivery of methotrexate through glucan particles. Adv Healthcare Mater. 2020;9:1901805.

86. Wileman TE, Lennartz MR, Stahl PD. Identification of the macrophage mannose receptor as a $175-\mathrm{kDa}$ membrane protein. Proc Natl Acad Sci U S A. 1986;83:2501.

87. Chu S, Tang C, Yin C. Effects of mannose density on in vitro and in vivo cellular uptake and RNAi efficiency of polymeric nanoparticles. Biomaterials. 2015;52:229-239.

88. Sun Q, Arif M, Chi Z, et al. Macrophages-targeting mannosylated nanoparticles based on inulin for the treatment of inflammatory bowel disease (IBD). Int J Biol Macromol. 2021;169:206-215.

89. Lautenschläger C, Schmidt C, Fischer D, et al. Drug delivery strategies in the therapy of inflammatory bowel disease. $A d v$ Drug Del Rev. 2014;71:58-76. 
90. Li W, Liu D, Zhang H, et al. Microfluidic assembly of a nano-inmicro dual drug delivery platform composed of halloysite nanotubes and a $\mathrm{pH}$-responsive polymer for colon cancer therapy. Acta Biomater. 2017;48:238-246.

91. Liu D, Zhang H, Cito S, et al. Core/shell nanocomposites produced by superfast sequential microfluidic nanoprecipitation. Nano Lett. 2017;17:606-614.

92. Oshi MA, Lee J, Naeem M, et al. Curcumin nanocrystal/ $\mathrm{pH}$-responsive polyelectrolyte multilayer core-shell nanoparticles for inflammation-targeted alleviation of ulcerative colitis. Biomacromolecules. 2020;9:3571-3581.

93. Oshi MA, Naeem M, Bae J, et al. Colon-targeted dexamethasone microcrystals with $\mathrm{pH}$-sensitive chitosan/alginate/Eudragit $\mathrm{S}$ multilayers for the treatment of inflammatory bowel disease. Carbohydr Polym. 2018;198:434-442.

94. Friesen DT, Shanker R, Crew M, et al. Hydroxypropyl methylcellulose acetate succinate-based spray-dried dispersions: an overview. Mol Pharm. 2008;5:1003-1019.

95. Bertoni $\mathrm{S}$, Liu Z, Correia $\mathrm{A}$, et al. $\mathrm{pH}$ and reactive oxygen species-sequential responsive nano-in-micro composite for targeted therapy of inflammatory bowel disease. Adv Funct Mater. 2018;28:1806175.

96. Deshpande S, Sharma S, Koul V, et al. Core-shell nanoparticles as an efficient, sustained, and triggered drug-delivery system. ACS Omega. 2017;2:6455-6463.

97. Naeem M, Bae J, Oshi MA, et al. Colon-targeted delivery of cyclosporine A using dual-functional Eudragit $\left({ }^{\circledR}\right)$ FS30D/PLGA nanoparticles ameliorates murine experimental colitis. Int J Nanomedicine. 2018;13:1225-1240.

98. Chen $\mathrm{L}$, You $\mathrm{Q}, \mathrm{Hu} \mathrm{L}$, et al. The antioxidant procyanidin reduces reactive oxygen species signaling in macrophages and ameliorates experimental colitis in mice. Front Immunol. 2018;8:1910.

99. Dagli Ü, Balk M, Yücel D, et al. The role of reactive oxygen metabolites in ulcerative colitis. Inflamm Bowel Dis. 1997;3:260-264.

100. Lee SH, Gupta MK, Bang JB, et al. Current progress in reactive oxygen species (ROS)-responsive materials for biomedical applications. Adv Healthcare Mater. 2013;2(6):908-915. doi:10.1002/ adhm. 201200423

101. Li S, Xie A, Li H, et al. A self-assembled, ROS-responsive janus-prodrug for targeted therapy of inflammatory bowel disease. J Control Release. 2019;316:66-78. doi:10.1016/j. jconrel.2019.10.054

102. Tapeinos C, Pandit A. Physical, chemical, and biological structures based on ROS-sensitive moieties that are able to respond to oxidative microenvironments. Adv Mater. 2016;28 (27):5553-5585. doi:10.1002/adma.201505376

103. Saravanakumar G, Kim J, Kim WJ. Reactive-oxygen-speciesresponsive drug delivery systems: promises and challenges. $A d v$ Sci. 2017;4(1):1600124. doi:10.1002/advs.201600124

104. Zhang Q, Zhang F, Chen Y, et al. Structure-property correlations of reactive oxygen species-responsive and hydrogen peroxideeliminating materials with anti-oxidant and anti-inflammatory activities. Chem Mater. 2017;29(19):8221-8238. doi:10.1021/ acs.chemmater. $7 \mathrm{~b} 02412$

105. Zhang D, Wei Y, Chen K, et al. Biocompatible reactive oxygen species (ROS)-responsive nanoparticles as superior drug delivery vehicles. Adv Healthcare Mater. 2015;4(1):69-76. doi:10.1002/ adhm.201400299

106. Li C, Zhao Y, Cheng J, et al. A proresolving peptide nanotherapy for site-specific treatment of inflammatory bowel disease by regulating proinflammatory microenvironment and gut microbiota. Adv Sci. 2019;6(18):1900610. doi:10.1002/advs.201900610

107. Zhang Q, Zhang F, Li S, et al. A multifunctional nanotherapy for targeted treatment of colon cancer by simultaneously regulating tumor microenvironment. Theranostics. 2019;9(13):3732-3753. doi: $10.7150 /$ thno. 34377
108. Coussens LM, Werb Z. Inflammation and cancer. Nature. 2002;420(6917):860-867. doi:10.1038/nature01322

109. Zhang Q, Tao H, Lin Y, et al. A superoxide dismutase/catalase mimetic nanomedicine for targeted therapy of inflammatory bowel disease. Biomaterials. 2016;105:206-221. doi:10.1016/j. biomaterials.2016.08.010

110. Patlevič P, Vašková J, Švorc $\mathrm{P}$, et al. Reactive oxygen species and antioxidant defense in human gastrointestinal diseases. Integr Med Res. 2016;5(4):250-258. doi:10.1016/j.imr.2016.07.004

111. Kim T-I, Rothmund T, Kissel T, et al. Bioreducible polymers with cell penetrating and endosome buffering functionality for gene delivery systems. $J$ Control Release. 2011;152(1):110-119. doi:10.1016/j.jconrel.2011.02.013

112. Wei X, Liao J, Davoudi Z, et al. Folate receptor-targeted and GSH-responsive carboxymethyl chitosan nanoparticles containing covalently entrapped 6-mercaptopurine for enhanced intracellular drug delivery in leukemia. Mar Drugs. 2018;16(11):439. doi: $10.3390 / \mathrm{md} 16110439$

113. Gou S, Chen Q, Liu Y, et al. Green fabrication of ovalbumin nanoparticles as natural polyphenol carriers for ulcerative colitis therapy. ACS Sustain Chem Eng. 2018;6(10):12658-12667. doi:10.1021/acssuschemeng.8b01613

114. Zhang S, Langer R, Traverso G. Nanoparticulate drug delivery systems targeting inflammation for treatment of inflammatory bowel disease. Nano Today. 2017;16:82-96. doi:10.1016/j. nantod.2017.08.006

115. Cheng S, Shen H, Zhao S, et al. Orally administered mesoporous silica capped with the cucurbit[8] uril complex to combat colitis and improve intestinal homeostasis by targeting the gut microbiota. Nanoscale. 2020;12(28):15348-15363. doi:10.1039/ D0NR03037F

116. Chhour P, Naha PC, O'Neill SM, et al. Labeling monocytes with gold nanoparticles to track their recruitment in atherosclerosis with computed tomography. Biomaterials. 2016;87:93-103. doi:10.1016/j.biomaterials.2016.02.009

117. Mohajeri M, Iranpour P, Vahidi Y, et al. Pegylated deoxycholic acid coated gold nanoparticles as a highly stable CT contrast agent. ChemistrySelect. 2020;5(29):9119-9126. doi:10.1002/ slct.202001634

118. Liu X, Zhang M, Yan D, et al. A smart theranostic agent based on Fe-HPPy@Au/DOX for CT imaging and PTT/chemotherapy/ CDT combined anticancer therapy. Biomater Sci. 2020;8 (15):4067-4072. doi:10.1039/D0BM00623H

119. Su Y, Liu S, Guan Y, et al. Renal clearable hafnium-doped carbon dots for CT/fluorescence imaging of orthotopic liver cancer. Biomaterials. 2020;255:120110. doi:10.1016/j.biomaterials.2020.120110

120. Truffi M, Sevieri M, Morelli L, et al. Anti-MAdCAM-1-conjugated nanocarriers delivering quantum dots enable specific imaging of inflammatory bowel disease. Int $J$ Nanomedicine. 2020;15:8537-8552. doi:10.2147/IJN.S264513

121. Eliakim R, Magro F. Imaging techniques in IBD and their role in follow-up and surveillance. Nat Rev Gastroenterol Hepatol. 2014;11(12):722-736. doi:10.1038/nrgastro.2014.144

122. Winklhofer S, Lin W-C, Wang ZJ, et al. Comparison of positive oral contrast agents for abdominopelvic CT. Am J Roentgenol. 2019;212(5):1037-1043. doi:10.2214/AJR.18.20445

123. Smith-Bindman R, Lipson J, Marcus R, et al. Radiation dose associated with common computed tomography examinations and the associated lifetime attributable risk of cancer. Arch Intern Med. 2009;169(22):2078-2086. doi:10.1001/archinternmed.2009.427

124. Naha PC, Hsu JC, Kim J, et al. Dextran-coated cerium oxide nanoparticles: a computed tomography contrast agent for imaging the gastrointestinal tract and inflammatory bowel disease. ACS Nano. 2020;14(8):10187-10197. doi:10.1021/ acsnano.0c03457 
125. Naha PC, Liu Y, Hwang G, et al. Dextran-coated iron oxide nanoparticles as biomimetic catalysts for localized and pH-activated biofilm disruption. ACS Nano. 2019;13 (5):4960-4971. doi:10.1021/acsnano.8b08702

126. $\mathrm{Hu} \mathrm{G}$, Guo M, Xu J, et al. Nanoparticles targeting macrophages as potential clinical therapeutic agents against cancer and inflammation. Front Immunol. 2019;10:1998. doi:10.3389/fimmu.2019.01998

127. Baldim V, Bedioui F, Mignet N, et al. The enzyme-like catalytic activity of cerium oxide nanoparticles and its dependency on $\mathrm{Ce} 3$ + surface area concentration. Nanoscale. 2018;10(15):6971-6980. doi:10.1039/C8NR00325D

128. Shi X, Yan Y, Wang $P$, et al. In vitro and in vivo study of $\mathrm{pH}$-sensitive and colon-targeting P(LE-IA-MEG) hydrogel microspheres used for ulcerative colitis therapy. Eur JPharm Biopharm. 2018;122:70-77. doi:10.1016/j.ejpb.2017.10.003
129. Pujara N, Wong KY, Qu Z, et al. Oral delivery of $\beta$-lactoglobulinnanosphere-encapsulated resveratrol alleviates inflammation in winnie mice with spontaneous ulcerative colitis. Mol Pharm. 2020;18(2):627-640. doi:10.1021/acs.molpharmaceut.0c00048

130. Wang X, Yan -J-J, Wang L, et al. Rational design of polyphenol-poloxamer nanovesicles for targeting inflammatory bowel disease therapy. Chem Mater. 2018;30(12):4073-4080. doi:10.1021/acs.chemmater.8b01173

\section{Publish your work in this journal}

The Journal of Inflammation Research is an international, peerreviewed open-access journal that welcomes laboratory and clinical findings on the molecular basis, cell biology and pharmacology of inflammation including original research, reviews, symposium reports, hypothesis formation and commentaries on: acute/chronic inflammation; mediators of inflammation; cellular processes; molecular mechanisms; pharmacology and novel anti-inflammatory drugs; clinical conditions involving inflammation. The manuscript management system is completely online and includes a very quick and fair peerreview system. Visit http://www.dovepress.com/testimonials.php to read real quotes from published authors. 